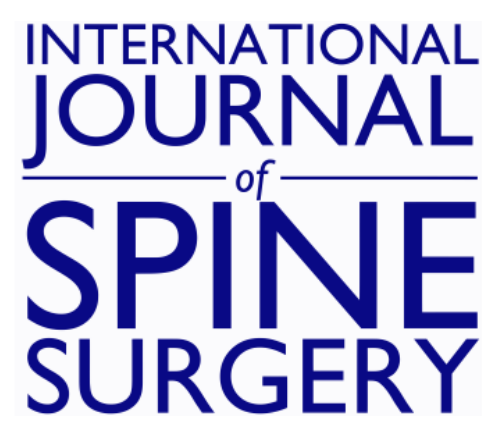

\title{
Sciatica: Detection and Confirmation by New Method
}

Satishchandra Gore and Sunil Nadkarni

Int J Spine Surg 2014, 8 ()

doi: https://doi.org/10.14444/1015

http://ijssurgery.com/content/8/15

This information is current as of April 26, 2023.

Email Alerts Receive free email-alerts when new articles cite this article. Sign up at: http://ijssurgery.com/alerts 


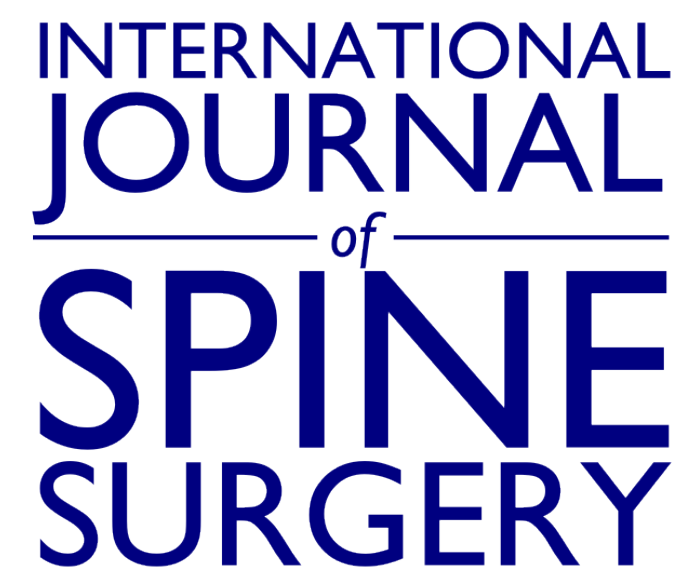

This article generously published free of charge by the International Society for the Advancement of Spine Surgery.

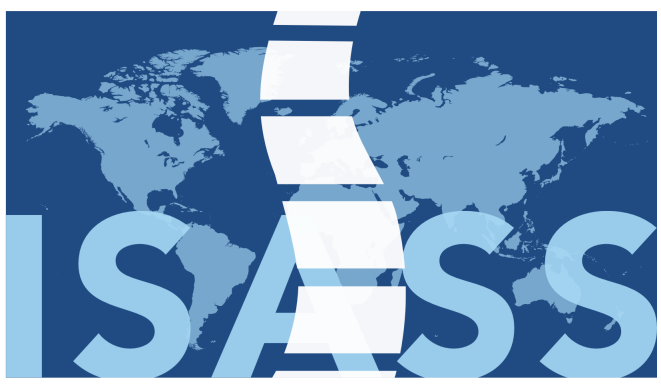

INTERNATIONAL SOCIETY for the ADVANCEMENT of SPINE SURGERY 


\section{Sciatica: Detection and Confirmation by New Method}

Satishchandra Gore, MS, FABMISS, ${ }^{1}$ Sunil Nadkarni, MS, FRCS, MCh ${ }^{2}$

${ }^{1}$ Prime Surgical Center, Pune, India ${ }^{2}$ Mangeshkar Hospital, Pune, India

\section{Abstract}

We need to overcome limitations of present assessment and also integrate newer research in our work about sciatica. Inflammation induces changes in the DRG and nerve root.It sensitizes the axons. Nociceptor is a unique axon. It is pseudo unipolar: both its ends, central and peripheral, behave in similar fashion. The nerve in periphery which carries these axons may selectively become sensitive to mechanical pressure-"mechanosensitized," as we coin the phrase. Many pain questionnaires are used and are effective in identifying neuropathic pain solely on basis of descriptors but they do not directly physically correlate nerve root and pain. A thorough neurological evaluation is always needed. Physical examination is not direct pain assessment but testing mobility of nerve root and its effect on pain generation. There is a dogmatic dominance of dermatomes in assessment of leg pain. They are unreliable. Images may not correlate with symptoms and pathology in about $28 \%$ of cases. Electrophysiology may be normal in purely inflamed nerve root. Palpation may help in such inflammatory setting to refine our assessment further.

Confirmation of sciatica is done by selective nerve root block (SNRB) today but it is fraught with several complications and needs elaborate inpatient and operating room set up. We have used the unique property of the pseudo unipolar axon that both its ends have similar functional properties and so inject along its peripheral end sodium channel blockers to block the basic cause of the mechanosensitization namely upregulated sodium channels in the root or DRG.

Thus using palpation we may be able to detect symptomatic nerve in stage of inflammation and with distal end injection, along same inflamed nerve we may be able to abolish and so confirm sciatica. Discussions of sciatica pain diagnosis tend to immediately shift and centre on the affected disc rather than the nerve. Theoretically it may be possible to detect the affected nerve by palpating the nerve and relieve pain moment we desensitize the nerve.

keywords:

sciatica, mechano sensitization, Inflammation, chemical, stretch sensitivity, pseudo unipolar axon, selective nerve root block, distal block, palpation of nerve

Volume 8 Article 15 - Endoscopic \& Percutaneous Special Issue doi: 10.14444/1015 


\section{Introduction}

Sciatica was thought to be due to pressure alone on the sciatic nerve root by a herniated disc. Newer understanding is chemical factors may be important in addition to mechanical factors. ${ }^{1}$ Nucleus pulposus can induce functional and structural nerve root changes in the absence of mechanical compression and this effect is mediated by substances located at the surface of NP cells. ${ }^{2,3,4}$ Radiculopathy in early stages is due to increased excitability and sensitization of DRG and root and results in projected pain, with pain very specifically along the distribution of the peripheral sensory nerve fibers. Excitability and sensitization of the fibers at that stage may respond to treatments directed at stabilizing oversensitive nerve membranes, by channel blockers, anesthetics and anticonvulsant medications. ${ }^{5,6,7,8,9,10,11}$ This nerve affection may progress to mechanical compression and nerve dysfunction in a linear way.

Primary afferent fibers have a unique morphology, called pseudo-unipolar, wherein both central and peripheral terminals have common axonal stalk. This distinguishes the primary afferent neuron from the typical neuron, where the recipient branch of the neuron (the dendrite) is biochemically distinct from the transmission branch (the axon). The biochemical functional similarity of central and peripheral terminals means that the nociceptor can send and receive messages from either end. This is a very significant functional characteristic. ${ }^{12}$

Neural tissue mechanosensitivity (local tenderness over nerve trunks and pain in response to limb movements that elongate the nerve) is a recognized feature of pain of neural origin .The straight leg raise (SLR) and slump tests are used to assess mechanosensitivity of the sciatic nerve tract. Nerve palpation is an additional assessment technique in the examination of neural tissue pain disorders. Under normal circumstances, peripheral nerve trunks are usually painless to non-noxious stimuli but if the nerve trunks are inflamed, even mild mechanical provocation, such as gentle palpation, can cause pain and protective muscle spasm. Therefore, if pain is provoked by the SLR and slump tests, then a similar pain response would be elicited by gentle nerve palpation. Traditionally nerves are palpated at back of thigh and knee and on lateral aspect of the knee, namely sciatic and tibial and common peroneal. In cases of sciatica commonly pain is felt below the knee so it may be necessary to palpate the nerves below the knee. This is to confirm mechanosensitization of the nerve all along its length.It is not known if in symptomatic patients with sciatica distal parts of the nerve are painful and tender or not. Palpation of nerve for tenderness in symptomatic patients is part A of our study.

Selective nerve root block (SNRB) in the foramen has been recently proposed as gold standard for diagnosing and managing leg pain. Use of steroids and ozone as antiinflammatory and local anesthetic as sodium channel blocker is done in SNRB. The injection may give relief of pain of variable duration and confirms origin of pain, by relieving it. Although the benefits for epidural steroid injections may include transient pain relief the risk attributed to these injections outweighs the benefits. It has been reported that injection along the course of painful sensitized but distal to area of affected nerve is also effective in relieving pain but it is not known where distally will it be most effective and what will be the mechanism of relief? This is part B of the study to see 
effect of sodium channel blocker on sciatic pain and mechanosensitization of the nerve when injected at distal most part of the nerve. We propose distal injection at the site of tender area found in symptomatic patient in part A of the nerve in cases of sciatica.

\section{Study Part A}

\section{Methods}

\section{Inclusion criteria}

Patients with back and leg pain in outpatient presenting within 3-12 weeks of their complaints, uninvestigated, and untreated. This was their first contact with a clinician for diagnosis and management of their pain and related complaints. Initially sciatic nerve around Ischial tuberosity, tibial nerve at popliteal fossa and or common peroneal nerve at or near fibular neck were palpated on symptomatic side and only if sciatic +1 of 2 were found to be tender, study was further refined by going more distal and detection of nerve ends or apparent ends which were tender was done. Choice of nerve to be palpated distal to knee was done based on apparent dermatomal distribution of pain. An attempt was done to correlate the tender part of the nerve with the apparent dermatome affected. Point of maximum tenderness of the nerve was noted. Since most patients were with pain distribution along L5 or S1 dermatome we palpated deep peroneal nerve and sural nerve respectively. The choice of the spot for palpation was dictated by ease of palpation of nerves against bony surfaces or ridges.

Point for palpation of deep peroneal nerve: Along the anterior aspect of ankle and then along upper ridge of calcaneus at sinus tarsi. Anatomically deep peroneal nerve in its distal part ends in a pseudo ganglion over roof of sinus tarsi and also ends in multiple branches to sinus tarsi and extensor digitorum brevis muscle.

Sural nerve: along lateral wall of calcaneus between lateral malleolus and tendo achillis. The sural nerve is subcutaneous and on lateral wall of calcaneus midway between tendo achillis and behind lateral malleolus.

Both these points were anatomically easy and were confirmed from anatomical books to be commonest part where these nerves will lay.

\section{Materials and results}

Study included consecutive 90 symptomatic sciatica patients. Forty-six had pain along the L5 dermatomal distribution and 34 had along S1. There was pain along both L5 and S1 areas in 18 of them. In L5 sciatica we found the lateral terminal branch of deep peroneal nerve was tender and very painful to touch. In case of S1 distribution pain it was found that sural nerve was very tender to palpate and touch. In symptomatic patients all had tenderness either on deep peroneal nerve or on sural nerve. Eighteen had tenderness on both nerves and were having pain along both L5 and S1 areas but one nerve may be more tender than other. The asymptomatic side was also palpated and was found tender in 4 (L5) and 7 (S1) patients. This examination was done as a part of routine clinical examination. 
Figure 1 shows the surface marking for the sciatic nerve and its branching at popliteal fossa. In initial step of the study nerve was plapated at near ischial tuberosity, and popliteal fossa and the neck of fibula. It was further palpated distal to knee on deep peroneal nerve as seen in Figure 2 and sural nerve as seen in Figure 3. The images above are from Grey's Anatomy and known to be royalty free. Author acknowledges the original images. 
A

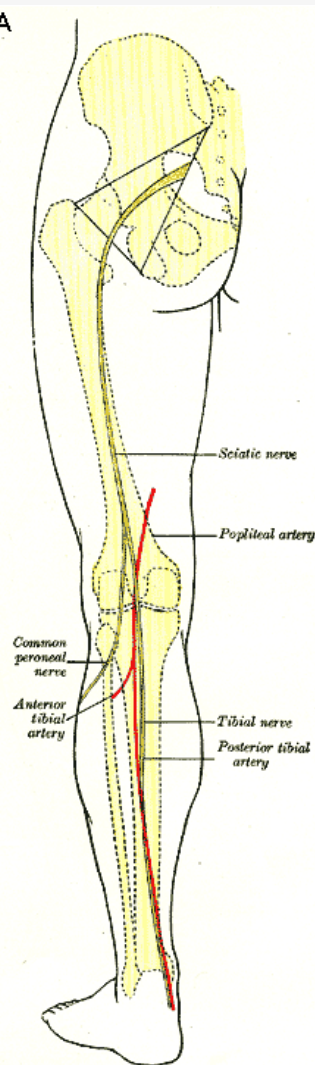

Fig. 1.

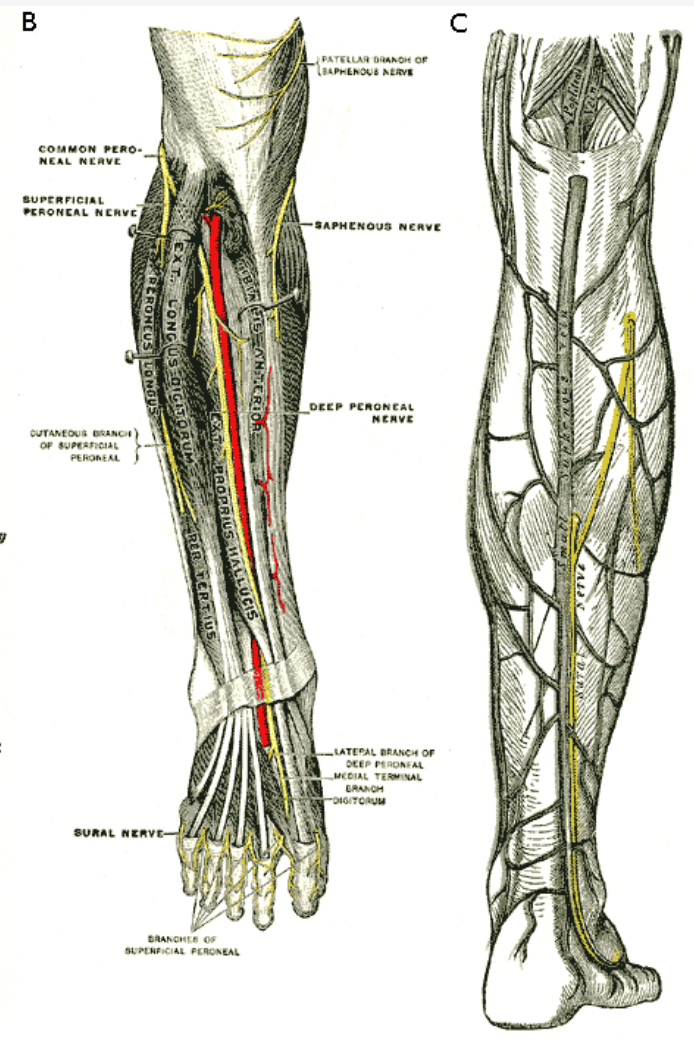

B

deal neave

supestic 


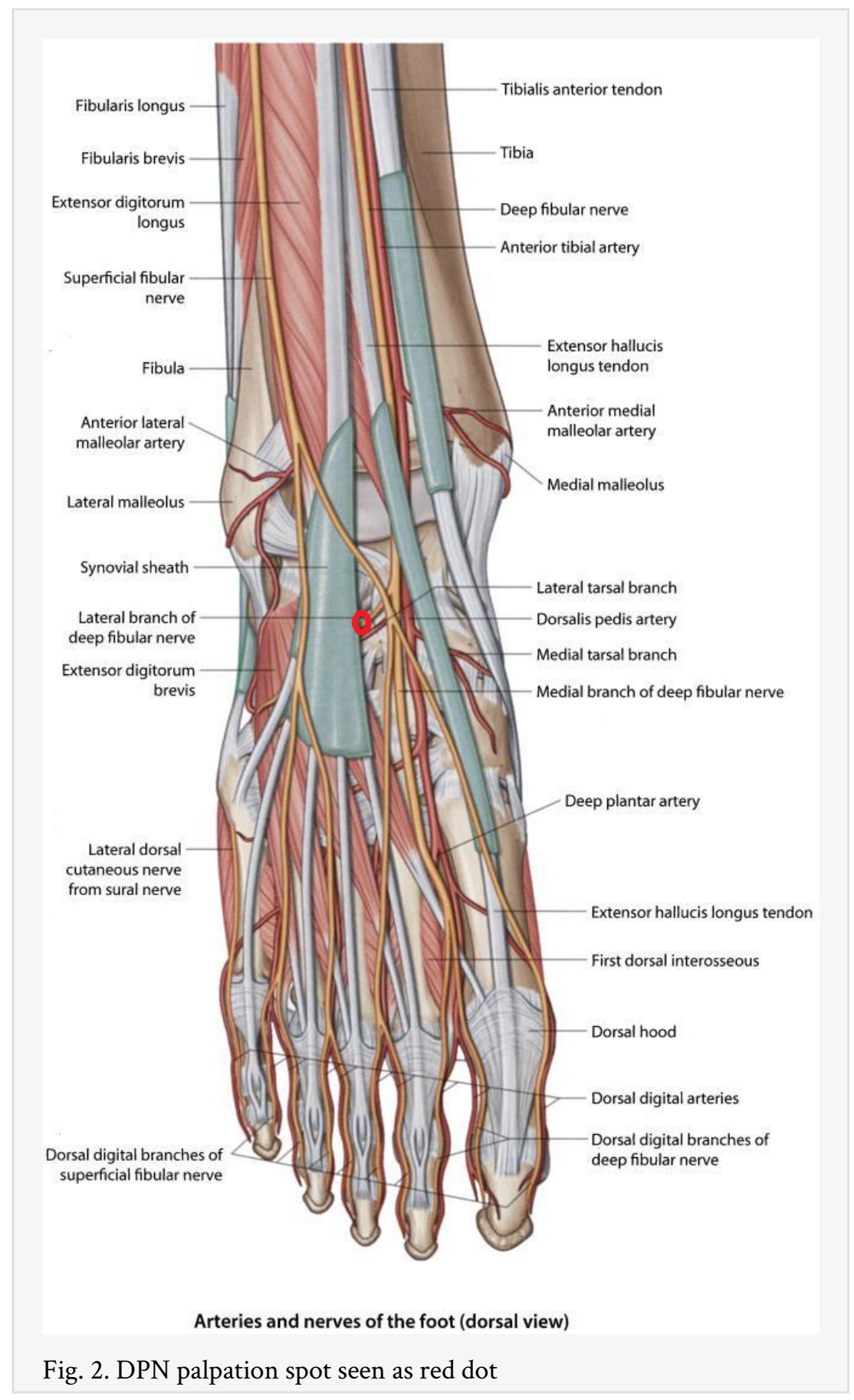




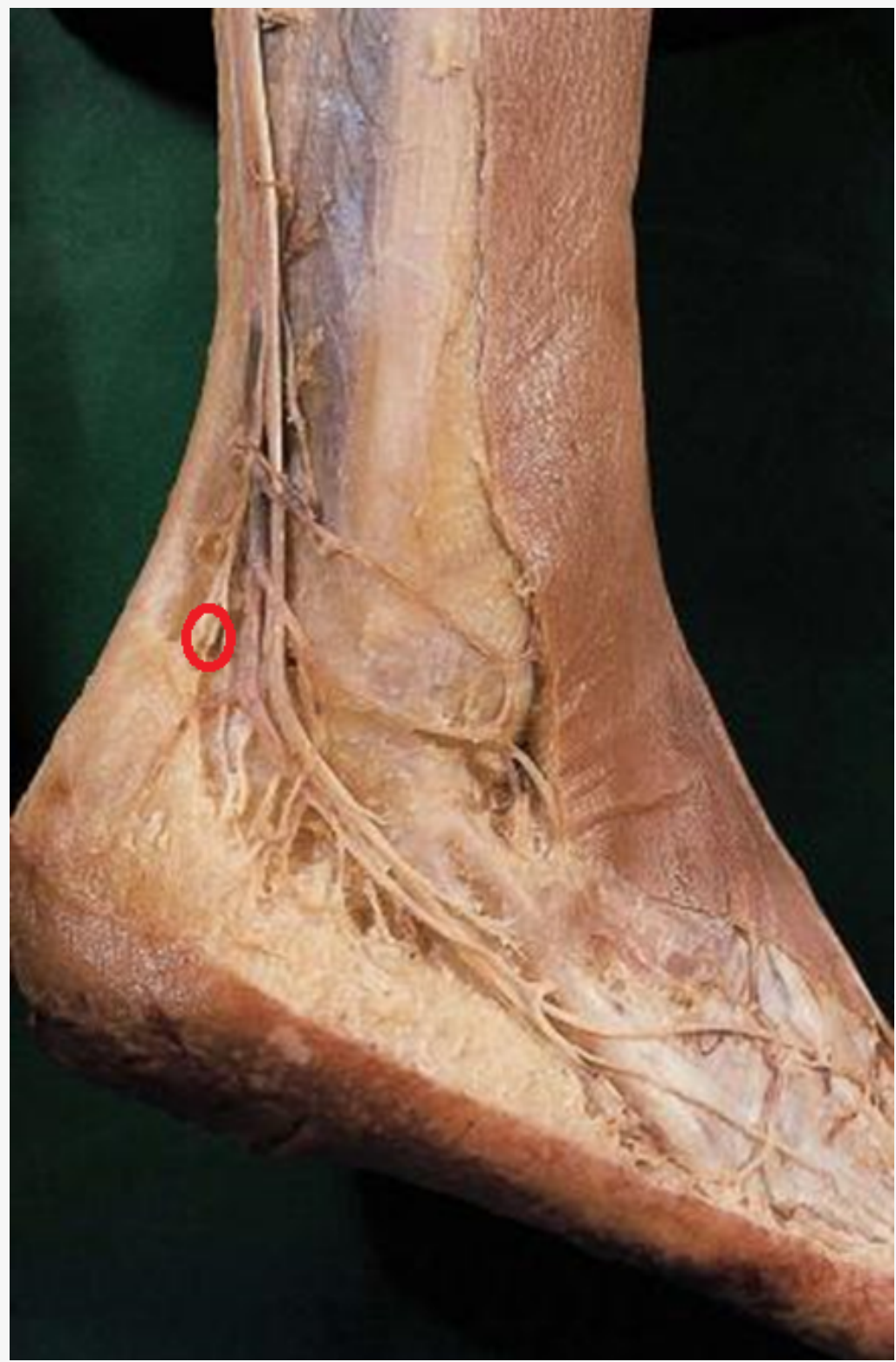

Fig. 3. Sural nerve in cadaver specimen in its constant location. BLUE dot signifies area to palpate against calcaneal wall.

\section{Study Part B}

In this part of the study injection of sodium channel blocker was given at identified tender nerve spot distally. The point chosen for injection was not empirical but it was the same tender point of the nerve end in symptomatic patient. Effect of the injection on the pain, and other clinical features and duration of relief was studied.

In case of deep peroneal it was along depth and walls of the sinus tarsi and for sural nerve it was along superior edge of the calcaneus at midway between tendoachilles and the lateral malleolus. The sinus tarsi area for injection is similar to Oconnor's area of sinus tarsi syndrome. 


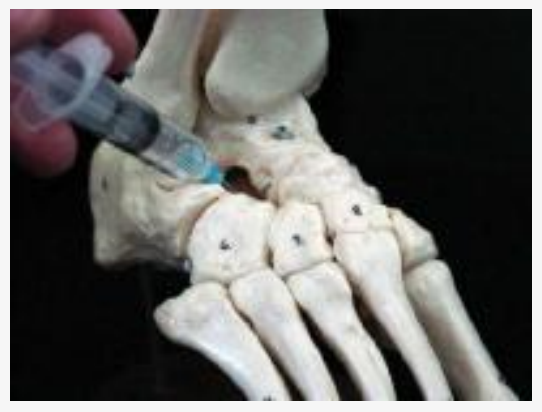

Fig. 4. Right DPN area

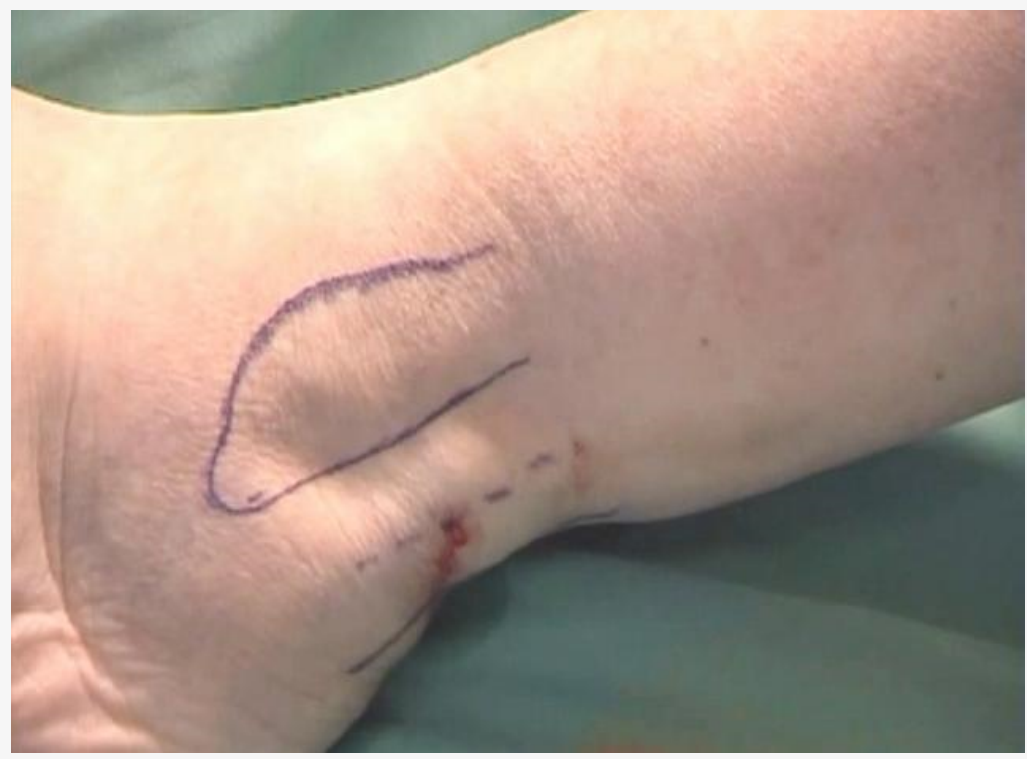

Fig. 5. Sural area for injection behind lateral malleolus.

\section{Materials}

Present injection study was done on 100 consecutive patients seen for back and leg pain in outpatient during January 2011 and December 2012. Written information about the study plan was given and explained to the patients highlighting the diagnosis and injection part. Informed Consent was taken, allergies noted.They presented with main complaint of leg pain and occasional additional back pain, or starting with back pain but now entirely leg pain alone. Patients who had back and leg pain, leg pain more than back pain, duration of 3 to 12 weeks of pain, on nonoperative care, patients positive SLR were included. They had no gross motor deficit but some had sensory affection ranging from tingling to hypoesthesia.

Exclusion criteria were patients already having a gross neurologic deficit. Postoperative status and presence of claudication also was criteria for exclusion. This excluded 33 patients.

We a had total (100-33) 67 patients of with age range 30-65; 38 were male and 29 were female. 


\section{Methods}

All patients were symptomatic with pain in leg along traditional L5 or S1 dermatome. Patient was assessed by routine history taking and assessment to rule out other red flags and local causes of pain. Standard neurological examination was done. Imaging study was routinely not ordered. Patient was asked to mark area of pain and it was noted if it fell in dermatome of L5 or S1. If the pain was predominantly in L5 area we utilized palpation of lateral terminal branch of deep peroneal nerve, for S1 sural nerve was palpated along lateral wall of calcaneus. The side of symptoms and duration noted. Once the nerve is found to be tender and painful on palpation we injected local anesthetic around end or distal identified painful area and noted its effect on the pain. We are injecting along distal part of the nerve and see its effect along the whole nerve trunk or tract and proximal part of back. Lignocaine $2 \%$ plain $3 \mathrm{cc}$ without adrenaline was injected .The injection was at one or both the spots depending on the presentation and location of symptoms. More painful side area was chosen. Effect on pain is noted at 10 minutes and 30 minutes. Relief of pain distribution was noted. Duration of relief noted. Effect on mobility of the back if affected was noted. Effect on knee mobility was noted.

\section{Results}

Patients meeting inclusion criteria cited as primary symptom pain distributed along the L5 dermatome with tenderness on palpation of the lateral terminal deep peroneal nerve (LTDPN) $(n=43)$ and pain distributed along S1 with tenderness in the sural nerve $(n=24)$ with some patients experiencing both $(n=13)$. Sixty-seven patients, therefore, showed 80 points of pain.

An injection of lignocaine was delivered to all pain points (50 at sinus tarsi for LTDPN; 30 at the lateral wall for sural nerve pain).

At 10 minutes postinjection, relief of pain is noted. Average VAS scores decreased from 7 preinjection to 1 for patients in the LTDPN group; for patients in the sural nerve treatment group, average VAS scores decreased from 8 to 2 . At 30 minutes posttreatment, no patients in the LTDPN group reported any pain; only 3 of the 24 patients in the sural nerve treatment group reported a VAS greater than 0 ( 1 in all cases).

Duration of relief averaged 8 hours (range 3-36 hours).

The mobility of the back improved with relief of pain. In patients who had pain and knee mobility affection, knee regained normal mobility.

In some patients $(n=13)$ both nerves were tender and pain distribution was patchy and more along both dermatomes. But more tenderness was noted on symptomatic side and also relevant to narration by patient, namely pain along back of the leg, calf, heel had sural tenderness more than DPN tenderness. All symptomatic patients had the nerve tender. All 67 patients responded to an injection of local anesthetic given at its distal end by relief of pain of varying duration. There were no untoward reactions to injection of lignocaine (plain without adrenaline and preservative). 


\section{Discussion}

Patient's main concern is pain and we need to increase our certainty about our diagnosis. Traditionally back related pain in leg has been diagnosed by history taking, noting pain descriptors, using questionnaires to rule out or confirm neuropathic pain, a proper relevant neurological examination and thinking based on dermatomes, use of judicious imaging, use of electrophysiological studies. All of the above have several limitations.

Focus on nerve root itself is lacking in studies of sciatica and nerve mediated leg pain. In our patients we were able to offer direct confirmation of nerve root involvement by palpation. In addition we were able to relieve the pain in very short time. The reverse action of distal injection can be explained by a unique property of the nociceptor.

We shift image or electro diagnosis paradigm to nerve root paradigm. Since this needs no technology its adaptation also is likely to be easy. Like a usual testing of peripheral pulses we need to promote peripheral nerve palpation as a part of usual testing for nerve root involvement. This would save lot of time money and efforts in clinical setting and also refine our clinical assessment in primary and specialty care related to early diagnosis of mechanosensitization of the nerve in symptomatic patients with sciatica.

In our study for the first time we have demonstrated that distal ends of deep peroneal nerve at lateral terminal branch and sural nerve are tender in symptomatic patients with L5 or S1 pain distribution. We also have 13/67 patients who have both nerves tender and needed injection for both the tender points. Sural nerve in its retro malleolar course is constant even though proximally it may have many variations. ${ }^{13}$ It is also significant to note that it is entirely sensory nerve.

It has been reported that leakage of chemical mediators or inflammatory cytokines, which are produced in the painful disc, into the epidural space through anular tears could lead to injury to nerve roots and may be primary pathophysiologic mechanism of radiating leg pain without disc herniation. Our study part A correlated nerve inflammation to pain by palpation of the nerve. Symptomatic nerve was demonstrated to be tender. For the first time the affected structure is identified clinically.

Degeneration in functional spinal unit is characterized by structural failure in its components. This is sensed and responded to by nervous system. ${ }^{14}$ It results in pain as main symptom. The intervertebral disc starts changing from age of 20 years. Diminished nutrition to the intervertebral disc in the first half of the second life decade appears to initiate tissue breakdown. ${ }^{15}$ The degenerative changes are mainly in form of annular tears.

It is likely that the disc may leak cytokines form the tears giving chemical radiculitis. ${ }^{16,17}$ It may manifest as pain due to chemical inflammation alone without mechanical compression. It can be cause of pain and its persistence. ${ }^{18}$ The concept of radiculitis was first described by Lewin in 1943, when he discussed the condition of irritation of the lumbar and sacral nerve roots. Recently role of Phospholipase A2 has been highlighted in inflammation in sciatica. ${ }^{19}$ Our focus is not identifying or staging degeneration but nerve affection and involvement. 
Pathophysiology of disc-related sciatica has strong chemical component. Sciatica in patients with disc disease was long ascribed to pressure on nerve root by a herniated disc. However, a role for chemical factors and mechanical insult is suggested by a number of clinical observations: large disc herniations are not always symptomatic, severe pain may be present in patients without imaging evidence of nerve root compression, the severity of symptoms and neurological signs is not well correlated with the size of the disc herniation, and conservative therapy is often effective, disc herniations can undergo spontaneous resorption and disc surgery does not consistently provide pain relief. ${ }^{20}$

The intervertebral disc is immunogenic, and mediators for inflammation have been identified within intervertebral disc tissue providing further evidence for the chemical cause. The current pathophysiological theory incriminates pro inflammatory substances secreted by the nucleus pulposus (NP). ${ }^{20}$ Changes of sodium channel density are known to be directly correlated to ectopic discharge rate. New hypothesis about pain states that pain is either due to inflammation or end result of inflammatory response. This is proposed as new law of pain. ${ }^{21}$ Pain is a state (of neural tissue) dependent sensory experience and there is a clear distinction between nociception and inflammatory pain. ${ }^{22,23}$ Sodium-channel dysfunction likely plays an important role in leading to ectopic generation of action potentials. The patient gets episodes of abrupt "pain attacks." These attacks may correspond to bursts of spontaneous activity in the injured afferents related to sodium-channel dysfunction. ${ }^{24,25}$ Nucleus pulposus on contact is known to induce inflammation and mechanosensitization in nerve root and DRG. The disintegrated DRG capsule is known to show increased permeability; a possible entrance route for various substances induced by locally applied nucleus pulposus. ${ }^{26}$ Large and small sensory afferent nerve fibers are affected in lumbar radiculopathy. ${ }^{27}$ Morphological changes in terms of an epidural inflammatory reaction and minor axonal and Schwann cell damage were demonstrated within $3 \mathrm{~h}$ of NP application, with or without compression. ${ }^{28}$ Peripheral input has been documented as necessary and sufficient for the persistence of several of these pain states implicating unresolved nociceptor sensitization. ${ }^{29}$ This sensitization can be detected by palpating the nerve. This sensitization probably is relieved by injection of the sodium channel blocker.

Primary afferent fibers have a unique morphology, called pseudo-unipolar, wherein both central and peripheral terminals emanate from a common axonal stalk. This distinguishes the primary afferent neuron from the prototypical neuron, where the recipient branch of the neuron (the dendrite) is biochemically distinct from the transmission branch (the axon). ${ }^{12}$ Literature mentions examples of dichotomizing axons to explain certain other clinically noted phenomena. Patient having groin pain and lower lumbar disc affection may have the pseudo unipolar dichotomizing axon as the basis. ${ }^{30}$ Both the peripheral and central terminals can be targeted by a host of endogenous molecules (such as $\mathrm{pH}$, lipids, and neurotransmitters) that regulate its sensitivity. Therapeutics directed at both terminals can influence the transmission of pain messages. Topically applied drugs (such as local anesthetics or capsaicin) can regulate pain via an action at the peripheral terminal. This has been amply shown in part B of our study. We have explained for the first time how a distal block can relieve proximal pain. 
We are concerned about the continued rise in disability associated with low back problems despite technological advances and greater expenditure on diagnostic tests and treatment. These have been mentioned time and again in social forums and political meets as well as scientific literature. The path to improvement lies in refining our approach to detection and confirmation of sciatica and monitoring it during its treatment till it is resolved.

Sciatica is mainly diagnosed by history taking and physical examination. The diagnostic value of history and physical examination has not been well studied. ${ }^{31}$ Physical examination alone today is totally irrelevant to newer understanding about pain and its mechanisms. History taken from patient may be related to cause, patients' perception about cause, and about location and severity of affect mainly pain or deficit. It may rule out trauma, infection and likely ischemia. Long duration and chronicity may add to affect. It is possible to differentiate nerve sensitization, denervation and central sensitization based on narration and examination. Pain related or symptom related descriptors may help in identifying neuropathic pain using Leeds Assessment of Neuropathic Symptoms and Signs (LANsS) ${ }^{32}$ We may also be able to segregate claudication from radicular sciatic pain. Our prime concern is to diagnose the involvement of nerve root or DRG in symptomatic patients. It is important that patients make sense of their suffering to tolerate it and evolve better. In clinical assessment since patient narration alone is not reliable for diagnosis and added examination may be more important than just narration. Examination may lead to precise localization of pain generator. But added confounding factors and medical co morbidities may make it less valuable. It helps us to rule out trauma, infection, and ischemia. Symptoms are dynamic and they cannot be captured in static examination. There is no refined clinical sign to detect inflammation in affected nerve during traditional examination. It is also important to note that many times it is difficult to distinguish L5 radiculopathy and peroneal nerve affection on traditional clinical examination alone. ${ }^{33}$

Pain may also change with time, posture and position. The pain in leg may become centralized on movement of extension and then is known to have a good correlation with annular integrity. McKenzie's sign elucidates its mechanism and it may be more dependable than an MRI. This is one sign which may be useful in absence of MRI access. It also highlights the nondependable nature of static dermatomal basis of diagnosing pain generator. The change in pain location in McKenzie's test points to annular integrity and function. ${ }^{34}$

In dermatomes we are trying to detect the skin affection due to involvement of the DRG or root. Traditionally dermatomes are a favored target in examination but they are old, nonspecific, nonreliable and too approximate. Sensory fibers are carried from DRG to spinal cord and to periphery. The nociceptors are unique and even though they are distributed in different formats they still give perception of pain. They are pseudounipolar axons. Due to their unique properties we have pain felt in periphery even though affection may be in centre. ${ }^{12}$ 
In rat study it has been found innervation territory of a lumbar dorsal root ganglion forms a cone, with the apex at the ganglion and the base circumference at the dermatome. As L5 or S1 DRG do not or only sparsely innervate the lumbar spine, so commonest radiculopathies of L5 or S1 levels do not cause pain sensed over the lumbar spine but cause pain in leg below knee. ${ }^{35}$

Symptoms may be outside classical dermatomal areas involving deeper tissues. The deeper tissues thus affected may be called dynatomes. ${ }^{36}$ The dermatomes are enunciated more than 100 years back without further refinement. The pain is not known to follow dermatomal patterns except in S1 in recent study. ${ }^{37}$ There is unnecessary dogma about dermatomes. They are old and unreliable even though we have recently come up with "evidence based dermatome maps" ${ }^{38}$ With known limitations of dermatomes which are thought to be the closest we go to knowing changed functioning of nociceptors we may have to look at shifting our focus from dermatomes to nociceptors "affected fibers with their unique properties." This may open up new possibilities in clinically identifying the affected DRG or root. ${ }^{39,40,41}$

In 1942 J. J. Keegan and F. D. Garrett reported on segmental distribution of cutaneous nerves in the extremities. Regular pattern of serial dermatomes in the limbs extending as continuous patterns from the dorsal midline of the body down the arms and legs are described. ${ }^{42}$ Kortelainen in 1985 identified limited utility of then existing maps so pain, neurological changes, $\mathrm{CT}$ and operative findings were correlated. S1 affection was seen even with L2-3 (1\%) L3-4(1.5\%) L4-5(34\%) and L5-S1(63\%) discs, perhaps because the traversing root was affected. Indicating that $\mathrm{S} 1$ dermatome can be affected by discs at many levels. The neurologic picture of high herniations was completely unreliable. ${ }^{43}$ Nitta in a 1992 study used SNRB in addition to symptom analysis. This is supposed to be best study about dermatomes and their correlation with skin manifestation and root affected. It was concluded that marked difference between L4, L5, and S1 may be caused by the diversity of the dorsal rami of the spinal nerves and double control by the lateral cutaneous nerve of the thigh. ${ }^{44}$ Direct correlation in skin and neural element is too simplistic. Inter segmental connections in dorsal spinal rootlets are known making dermatomes more unpredictable. It is also important to note that dermatome is area of skin innervated by particular nerve root, DRG or spinal segment and they are not peripheral nerve fields. Lee has described the best available map by integration of all available evidence. In this map the overlap of C7 and L5 is acknowledged. ${ }^{39}$ In our study we have directly correlated pain with the nerve physically and have been able to relieve the symptoms by utilizing same tender area for blocking involved sodium channels.

SLR and slump test are not positive in absence of compression of nerve root. Slump test is sensitive and SLR is more specific for herniation with root compression. In absence of mechanical compression and affection of root mobility the SLR may be normal. There are many patients who have severe pain along the leg but SLR may be negative. We are clueless in these patients where present study may shed light.

MRI is taken as the gold standard for nerve root involvement assessment but it is less sensitive than clinical examination. Imaging studies may need contrast studies, dynamic studies, and concepts about instability in images are still not reliable. MRI-visible nerve involvement is significantly less common as compared to detection by physical examination and pain drawing findings of nerve involvement in corresponding body 
segments. ${ }^{37,45}$ As most issues and decisions about treatment and outcomes today depend on MRI nerve root involvement assessment than clinical detection; we need to make clinical examination more meaningful and dependable by overcoming disconnect and paradox in images and symptoms.

Patients with anular tears may experience low back pain or radiation of pain into the lower extremity. Chemical radiculitis is an inflammatory condition of the nerve root, which may result from the rupture of the anulus fibrosus and dissemination of disc fluid along the nerve root sheath. ${ }^{25}$ In some patients discography solves this puzzle when we have leak of the dye from an annular tear and its flow along root sleeve that causes pain or gets relieved on analgesic discography. CT scan may be used as an adjunct to discography. ${ }^{46}$ Provocative discography is one of the methods for diagnosing discogenic chemical radiculitis. Symptoms of extremity pain, MR imaging void of local nerve root pathology, an electromyography study positive for an acute radiculopathy, and a positive fluoroscopically guided diagnostic selective nerve root block make a diagnosis of chemical radiculitis. However, morphologic evaluation of the nerve root caused by chemical radiculitis is not practised. In our present study we have demonstrated that an inflamed root is tender to palpate in part A and its involvement can be confirmed by injecting sodium channel blocker along its distal end in part B.It may be possible to use 3D MRI for chemical radiculitis detection. All patients with radicular leg pain caused by chemical radiculopathy showed nerve root swelling on 3D MR Radiculography. ${ }^{47}$ Symptoms noted in structured physical examination and pain diagram may be caused by MRI invisible factors. Inflammation may be detected in MRI by use of additional imaging with enhancement; but may not be feasible in routine assessment. The MRI invisible factors need treatment directed at nerve root involvement. We need to understand these MRI invisible factors to overcome this problem situation. Lack of precision in assessing nerve involvement may result in inadequate and improper analgesia treatment and dissatisfaction in patients' mind and our inability to prognosticate. ${ }^{37}$

Today in absence of any sign to monitor peri neural inflammation and resultant chemical sciatica; the progress on follow up or during conservative or surgical treatment is assessed by just narration of the patient about intensity on a VAS scale or oswestry disability index. There is no objective assessment. In symptomatic patients MRI may be helpful but Images may not correlate with pain generation cause and process in about $28 \% .{ }^{45} \mathrm{In}$ postoperative cases to monitor the case serial MRI has been advised but cost considerations make it difficult.Recent advances in magnetic resonance (MR) imaging of nerves has ensured that MR neurography is capable of providing information about nerve compression, nerve inflammation, and recovery of nerve from pathological states. However, MR Neurography has yet to be used to correlate these findings with mechanosensitivity. ${ }^{48}$ Neurography may be more useful than electro diagnosis in cases of radiculopathy. ${ }^{49}$ However, nerve-root inflammation is a key feature of sciatica, and the state of inflammation may be more important than anatomic contact between disc and nerve root. ${ }^{50}$ Discerning the clinical relevance of spine imaging results is an ongoing challenge. However we can avoid imaging entirely in situations in which it is unlikely to be useful. ${ }^{51}$ In such situations our ability to palpate and detect a sensitized nerve is more important. If we are able to relieve the pain in real time, it has significant positive impact on patients. 
It has been pointed out that spine imaging is not really contributory to diagnosis and may not change outcomes. Reporting of imaging also needs to be presented to a patient with all caveats. ${ }^{52}$ Real-time ultrasound has been used in the investigation of pain disorders in the upper limb and sciatic nerve movement in the lower limb. However, at this stage, the usefulness of this procedure may be restricted to measurement of nerve movement rather than mechanosensitivity. ${ }^{53}$ Thus imaging is not enough for nerve root involvement assessment in a mechanosensitized nerve in symptomatic patient.

Electrophysiological studies may report normal results even in painful conditions, positive only if physiological integrity of nerve is affected. This test may make us aware of any collateral damage in nervous system. They essentially are not for diagnosing pain. ${ }^{54}$ It is likely that pain in the leg which may be nerve mediated may be missed.

Minimally invasive endoscopic visualization of pain generators is possible. During these surgeries we have been able to manipulate the DRG and nerve root and appreciate the pain perception in certain area by the patients. These areas vary as per area of the root probed, root axilla, body or outer border. ${ }^{55}$ Yeung and Gore have reported endoscopic in vivo visualization of pain generators and their treatment. We can palpate inflamed nerve at foramen which may induce pain along its peripheral distribution.

Neural tissue mechanosensitivity (local tenderness over nerve trunks and pain in response to limb movements that elongate the nerve) is a recognized feature of pain of neural origin. Nerve palpation has been advocated as an additional assessment technique in the examination of neural tissue pain disorders. Under normal circumstances, peripheral nerve trunks are usually painless to non-noxious stimuli. However, if the nerve trunks are inflamed, even mild mechanical provocation, such as gentle palpation, can cause pain and protective muscle responses. Therefore, if the sciatic nerve tract is sensitized and pain is provoked by the SLR and slump tests, then a similar pain response is elicited by gentle nerve palpation. Increased sensitivity of nerve trunks to palpation is as a result of inflammation. In one study, ${ }^{54}$ reliability, validity and diagnostic accuracy of manual palpation of the sciatic, tibial and common peroneal nerves in the examination of lowback related leg pain was examined. But nerves were palpated in only above knee location. They were not palpated below knee and in distal area of the lower limb. This needs refinement.

In previous reported studies only proximal nerve trunks were palpated and palpation was validated for diagnosing nerve root mechanosensitization. We have further refined this palpation to focus on distal end of the nerve. This focus was prompted by idea that since the inputs for pain in these cases may be from periphery we need to go to distal most points of the nerve which is likely to be affected. As it is distribution of L5 and S1 does extend upto toes. Once we have used the distalmost part we will be able to integrate affection of the full length of root or axons all along their length. We have successfully correlated nerve with dermatome.

The presence of increased neural tissue mechanosensitivity identified in part by nerve palpation is one of the key factors in the classification of low-back related leg pain. Classification of patients with back related leg pain into subgroups with targeted intervention, has been suggested as a mean of improving treatment outcome and there is preliminary evidence to support this. ${ }^{56}$ Although the SLR and slump tests are tests of 
"stretch" mechanosensitivity, palpation may be seen as a sign of "pressure" mechanosensitivity. ${ }^{57,58}$ In our routine examination we can integrate palpation. In view of the finding that commonly sciatica is present distal to knee we must palpate both common peroneal and its branches and sural nerve. We have used above described two spots as preferred areas for manipulations related to L5 and S1 roots. We have consistently found these two spots very tender indicating an inflammation base in symptomatic patients with L5 or S1 sciatica. ${ }^{59}$

\section{Selective Nerve Root Block}

It has been shown that history, physical examination, imaging, and nerve conduction studies in non-radicular or discogenic pain are unable to diagnose the precise cause in $85 \%$ of the patients. In contrast, controlled diagnostic blocks have been shown to determine the cause of pain in as many as $85 \%$ of the patients. Level of evidence for selective nerve root blocks for diagnostic purposes is Level II-III ${ }^{60}$ In patients with subacute or chronic radicular symptoms, there is good evidence that a single TFESI has similar efficacy as a single transforaminal injection of bupivacaine or saline. Literature supports their use in the treatment of lumbosacral radicular pain. ${ }^{61}$ The popularity of neural blockade as a diagnostic tool in painful disorders is specifically because it helps to determine pathophysiology of clinical pain, site of nociception and inflammation, pathway of afferent signals. Information gained then may be applied to choice of medicines, therapeutic blocks and surgery, and used to anticipate response to neuroablative therapies. Injections around spinal nerves alone are not dependable for decision making in neuroablative procedures. ${ }^{62} \mathrm{SNRB}$ is the gold standard today as it is affect related and can detect pain generator, but needs elaborate set up, stay in hospital and fought with severe complications. Although the benefits for epidural steroid injections may include transient pain relief for those with/without surgical disease, the multitude of risks attributed to these injections outweighs the benefits. In one study On the basis of a definition of a positive block as $70 \%$ pain relief, it had a sensitivity of $57 \%$, a specificity of $86 \%$, an accuracy of $73 \%$, a positive predictive value of $77 \%$, and a negative predictive value of $71 \%$. False-negatives were due to insufficient infiltration, insufficient passage of the injectate, and intraepineural injections, false-positives resulted from overflow of the injectate from the injected asymptomatic level into either the epidural space or symptomatic level. ${ }^{63}$

Complications of SNRB are due to contaminated epidural steroid injections resulting in meningitis, stroke, paralysis, and death. The Center for Disease Control (CDC) specifically identified 25 deaths (many due to Aspergillosis), 337 patients sickened, and 14,000 exposed to contaminated steroids. Nevertheless, many other complications go unreported underreported: Other life-threatening infections, spinal fluid leaks (0.4-6\%), positional headaches (28\%), adhesive arachnoiditis (6-16\%), hydrocephalus, air embolism, urinary retention, allergic reactions, intravascular injections (7.9-11.6\%), stroke, blindness, neurological deficits/paralysis, hematomas, seizures, and death. ${ }^{64}$

In recent editorial in Spine65 the utility of SNRB in diagnosing is considered negative. In essence, it posits that SNRBs are unable to diagnose spinal pathology; SNRB pain relief may be due to blockade of the indirect contributors to pain or placebo; and concludes that, given concerns about safety and their lack of diagnostic accuracy, SNRBs via the classic approach should no longer be performed and that the Kambin triangle approach is an 
improvement compared with the classic approach--the only role for SNRBs is therapeutic. We would like to propose distal block as a anatomically selective and physiologically mechanism specific block for diagnosis of sciatica.

There is limited evidence for selective nerve root injections as a diagnostic tool in evaluating low back pain with radicular features. ${ }^{66}$ Based on current weak evidence, it is unlikely that SNRB is a cost-effective method for identifying the symptomatic nerve root prior to lumbar spine surgery. ${ }^{67}$ It is possible that we may be able to give relief of radicular pain by distal block but at almost zero cost. This point needs further study by comparison of nerve root block proximal end compared with distal end with special reference to costs. A proper titrated dose of injectate may give long term relief.

Studies in older literature about distal to foramen blocks were done in an era when concepts about pain generation and unique properties of nociceptors were not refined. Studies were not able to add to the then state of art. Case series has been published as old as in 1960 where distal to lesion block was studied to relieve pain from a large area supplied by the nerve by blocking the afferent from that area. "Evidence that local anesthetic injections of the afferent pathway, distal to the site of the lesion, may stop the pain or paraesthesia that may far outlast the duration of the anesthesia; and blocking a peripheral nerve supplying a large part but not the whole of the region where the pain or paraesthesia are felt may remove these sensations from the entire region." ${ }^{68}$ No explanation as to the mechanism was given and was not suggested. Another study by Xavier et al highlights peripheral inputs as a cause of pain during distal block study. ${ }^{69,70}$

The effectiveness of common peroneal nerve block for lumbar disc herniation was evaluated in a double blind study by Tajiri et al. ${ }^{71}$ Common peroneal nerve block was performed near the fibular head in nine patients using $2 \%$ lidocaine and in 10 patients using saline. The average pain scale score decreased from 3.1 to 0.6 in the lidocaine group, whereas it decreased from 3.0 to 2.6 in the placebo group. The average result in the straight leg raising test increased from 61 degrees to 84 degrees in the lidocaine group, but from 44 degrees to 50 degrees in the placebo group. Lower leg pain lessened more in the lidocaine group than in the placebo group. The paper concluded lower leg pain disappeared or decreased with a lidocaine block at a site distal to the lumbar lesion suggesting impulses that are transmitted distal to the lesion may be important for the generation of sciatic radicular pain. In unreported study we have compared injections of local anesthetic with sodium chloride and it was found that local anesthetics were superior in giving relief of pain.

In a recent report in spine journal about disc related sciatica emphasis is added to shift our focus and thinking ${ }^{72}$ from disc herniation to nerve root involvement. Most sensitive and specific physical examination tests for the evaluation of radicular pain are conceptually based on the detection of neural tension (SLR) or nerve root dysfunction (sensorimotor deficits) and are only indirectly related to the disc herniation itself. Therefore disc herniation as reference standard is questionable and inappropriate. We should do all in our capacity to understand, detect, confirm, and monitor nerve root involvement irrespective of the pathological cause. We have done this in present study by proposing palpation of the nerve mainly to detect its increased mechanosensitivity. Pain relief even with distal 
block can be a replacement for transforaminal selective nerve root block and a confirmation of nerve mediated pain. This would then bring in nerve root affect paradigm in place of disc and related electro diagnostic or MRI imaging paradigm.

Saal et al. ${ }^{73}$ have emphasized accuracy, safety and reproducibility in clinical testing. The tests used to diagnose the source of a patient's pain require accurate determination of the abolition or reproduction of the patient's painful symptoms. We have been able to achieve abolition of pain in all patients even though for a limited period of time by injecting distally. We have also observed that SLR improved in patients partially. This partial improvement may be proportionate to component of inflammation than mechanical compression cause. In patients when SLR improved totally it is proposed that patient had apparently only inflammation as cause.

There is a fundamental question in musculoskeletal system and specifically in back and related leg pain. Where is the pain coming from? The study by kuslich et al answers that question. ${ }^{74}$ Sciatic nerve can be only painful if the root is inflamed and then mechanically stretched or compressed. Inflammation is a prerequisite for pain. Studies have extensively reported on effects of nucleus pulposus on nerve root morphology, sodium channels etc. Chen et al.'s study is remarkable in describing minute changes in axon morphology and its mechano sensitization and up regulation of sodium channels. This study describes a finer correlation between sodium channel levels and the nerve function. It nearly forms the basis of present study. ${ }^{75}$ Olmarker et al have studied and extensively published on effects of nucleus pulposus on nerve root in acute and chronic stages. ${ }^{76,77,78,79}$

Adjacent double root contribution is seen in unilateral sciatica. It has been reported recently in more than $50 \%$ cases. $^{80}$ it may make clinical presentation and assessment more confusing. In our study we found 13 patients had both nerves tender thus correlating with double root contributions. Both nerves on injection responded with relief of pain. We also are aware that level of sodium channels in related nerve will decide the dose of injectate of blocker but we have used the same $3 \mathrm{ml} \%$ lignocaine in all patients. Using different concentration and volume may be more or less effective. It may be possible to use other long acting anesthetics.

Positive results on injection and relief confirm diagnosis of sciatica as nerve-mediated pain. We confirm the mechanism of this pain which may be upregulated sodium channels in nerve by inducing relief using sodium channel blocker. This use of lidocaine for relieving effects of nucleus pulposus is well supported in literature. ${ }^{81}$

In previous reports of distal blocks the mechanism of its effectiveness has been unknown, site of injection also was not precise. Our study has been a advancement on both the counts of palpation and injection at distal end of the nerve.

We are able to relieve pain of radicular sciatica by transforaminal or epidural injection of steroids or local anesthetics at proximal end. This study is the first of its kind in eliciting this unique property of the nociceptor. It has always been traditionally thought that a proximal injection at intervertebral foramen around the root will relieve distal pain but here we are able to achieve same results by injecting the distal most portion of the nerve root. This is easy and needs no technology and is an office procedure. 
The idea of using local anesthetic came from its action a sodium channel blocker. Local anesthetic in addition has been used systemically and intravenous for relief of neuropathic pain. ${ }^{82}$ It is observed that decrease of neuropathic pain following local or systemic use of a local anesthetic is related to decrease of ectopic ongoing activity in injured afferent nerve fibers. It may be possible to relieve pain by using lignocaine patches locally over the affected nerve. We feel a further study is warranted to study this possibility.

Previous reports have highlighted mechanism-based classification of pain. ${ }^{83,84} \mathrm{We}$ feel that by our study we have integrated the research information in bedside clinical testing.

In a symptomatic patient who is MRI negative or has no significant findings in MRI instead of doing multiple tests it may be prudent to relieve the pain by a distal block as above and then analyze the likely cause and plan treatment. It is likely that if pain is totally relieved for a long duration, inflammation may be forming a dominant part of the symptom generation and non-operative care may be appropriate for the patient. In resource poor settings and where an access to MRI is not available this clinical test may be helpful. It is likely that we may have this as a clinical correlate for nerve mediated pain.

We would also like to study further and confirm the dermatome and tender nerve relation. The question of distal block needs further refined study as we will have to include all patients who may have spots of pain, and pain of non-traumatic and non-infective causes. This also may include patients with referred pain.

We are not proposing this "Gore sign" as treatment of nerve root involvement, but a precise diagnosis which may hold more promise. It is likely that with inclusion of nerve palpation in physical examination in addition to pain diagram, electro diagnosis, and image analysis we may be able to refine our diagnosis. We are aware that this is a simplistic view of the pain mechanisms and there are many approximations in this study. It is simple and has no technology barrier. It has worked in hands of the author. It overcomes all legacy limitations of the present day approach and most of its basis is validated.

Increasing certainty in treatment outcome may improve compliance by the patients. It may also be possible to predict that surgical intervention may not be needed in absence of mechanical compression and confirmation of chemical as the only cause. The author proposes this as the "Gore sign and block" and a new clinical correlate for chemical sciatica.

\section{References}

1. Incision of the Anulus Fibrosus Induces Nerve Root Morphologic, Vascular, and Functional Changes: An Experimental Study Kayama, Satoru MD; Konno, Shinichi MD, PhD; Olmarker, Kjell MD, PhD; Yabuki, Shoji MD, PhD; Kikuchi, Shinichi MD, PhD Spine: 15 November 1996 - Volume 21 - Issue 22 - p 2539-2543

2. Mechanical and biochemical injury of spinal nerve roots: a morphological and neurophysiological study M. Cornefjord, K. Olmarker, B. Rydevik, C. Nordborg European Spine Journal June 1996, Volume 5, Issue 3, pp 187-192 
3. Application of Nucleus Pulposus to L5 Dorsal Root Ganglion in Rats Enhances Nociceptive Dorsal Horn Neuronal Windup J. M. Cuellar, P. X. Montesano , J. F. Antognini , E. Carstens Journal of Neurophysiology Published 1 July 2005Vol. 94no. $35-48$

4. Effect of Nucleus Pulposus on the Neural Activity of Dorsal Root Ganglion Takebayashi, Tsuneo MD, PhD; Cavanaugh, John M. MS, MD; Cüneyt Özaktay, A. MD; Kallakuri, Srinivasu MS, and; Chen, Chaoyang MD Spine: 15 April 2001 Volume 26 - Issue 8 - pp 940-944

5. The effects of epidural application of allografted nucleus pulposus in rats on cytokine expression, limb withdrawal and nerve root discharge Srinivasu Kallakuri, Tsuneo Takebayashi, A. Cuneyt Ozaktay, Chaoyang Chen, Shangyou Yang, Paul H. Wooley, John M. Cavanaugh European Spine Journal December 2005, Volume 14, Issue 10, pp 956-964

6. Tumor necrosis factor alpha and nucleus-pulposus-induced nerve root injury. Olmarker K and Larsson K. Spine 23: 2538-2544, 1998.

7. Changes in spontaneous behavior in rats exposed to experimental disc herniation are blocked by selective TNF-alpha inhibition. Olmarker K, Nutu M, and Storkson R. Spine 28: 1635-1641, 2003.

8. Selective inhibition of tumor necrosis factor-alpha prevents nucleus pulposus-induced thrombus formation, intraneural edema, and reduction of nerve conduction velocity: possible implications for future pharmacologic treatment strategies of sciatica. Olmarker K and Rydevik B. Spine 26: 863-869, 2001.

9. Edema formation in spinal nerve roots induced by experimental, graded compression. An experimental study on the pig cauda equina with special reference to differences in effects between rapid and slow onset of compression. Olmarker K, Rydevik B, and Holm S. Spine 14: 569-573, 1989.

10. Autologous nucleus pulposus induces neurophysiologic and histologic changes in porcine cauda equina nerve roots. Olmarker K, Rydevik B, and Nordborg C. Spine 18: 1425-1432, 1993.

11. Pathogenesis of sciatic pain: a study of spontaneous behavior in rats exposed to experimental disc herniation. Olmarker K, Storkson R, and Berge OG. Spine 27: 1312-1317, 2002.

12. Cellular and Molecular Mechanisms of Pain Allan I. Basbaum et al. Cell. 2009 October 16; 139(2): 267-284.

13. Anatomy of the sural nerve: cadaver study and literature review. Riedl O1, Frey M. Plast Reconstr Surg. 2013 Apr;131(4):802-10

14. Pathology and possible mechanisms of nervous system response to disc degeneration. Brisby H. J Bone Joint Surg Am. 2006 Apr;88 Suppl 2:68-71.

15. The back pain revolution. Waddell G. London: Churchill Livingstone; 2004.

16. Epidemiology and natural history of low back pain. Eura Medicophys. 2004;40:9-13. [PubMed] Dunn KM, Croft PR.

17. The importance of radiating leg pain in assessing health outcomes among patients with low back pain. Results from the Veterans Health Study. Spine. 1998;23:470-474. Selim AJ, Ren XS, Fincke G, Deyo RA, Rogers W, Miller D. et al.

18. Clinical decision rules for identification of low back pain patients with neurologic involvement in primary care. Haswell K, Gilmour J, Moore B. Spine (Phila Pa 1976). 2008 Jan 1;33(1):68-73. 
19. Effects of Phospholipase A2 on Lumbar Nerve Root Structure and Function Chen, Chaoyang MD; Cavanaugh, John M. MD; Ozaktay, A. Cuneyt MD; Kallakuri, Srinivasu MS; King, Albert I. PhD Spine: 15 May 1997 - Volume 22 - Issue 10 - p 1057-1064

20. Pathophysiology of disk-related sciatica. I.-Evidence supporting a chemical component Denis Mullemana, b, Saloua Mammoub, Isabelle Griffoulb, Hervé Watiera, Philippe Goupillea, Joint Bone Spine Volume 73, Issue 2, March 2006, Pages 151-158 Review

21. The biochemical origin of pain - Proposing a new law of pain: The origin of all pain is inflammation and the inflammatory response. Medical Hypotheses (2007) 69, $70-82$

22. Transcriptional and posttranslational plasticity and the generation of inflammatory pain CLIFFORD J. WOOLF AND MICHAEL COSTIGAN Proc. Natl. Acad. Sci. USA Vol. 96, pp. 7723-7730, July 1999 Colloquium Paper

23. Pain Mechanisms: A Commentary on Concepts and Issues Edward R. Perl Prog Neurobiol. 2011 June ; 94(1): 20-38. doi:10.1016/j.pneurobio.2011.03.001

24. Mechanisms of Neuropathic Pain James N. Campbell, and Richard A. Meyer Neuron. 2006 October 5; 52(1): 77-92.

25. Assessment and Management of Acute Low Back Pain ROBERT L. BRATTON, M.D., Mayo Clinic Jacksonville, Jacksonville, Florida Am Fam Physician. 1999 Nov 15;60(8):2299-2306.

26. Incision of the Intervertebral Disc Induces Disintegration and Increases Permeability of the Dorsal Root Ganglion Capsule Murata, Yasuaki MD, PhD†; Rydevik, Björn MD, PhD; Takahashi, Kazuhisa MD, PhD; Larsson, Karin BSc; Olmarker, Kjell MD, PhD Spine: 1 August 2005 - Volume 30 - Issue 15 - pp 1712-1716

27. The Function of Sensory Nerve Fibers in Lumbar Radiculopathy: Use of Quantitative Sensory Testing in the Exploration of Different Populations of Nerve Fibers and Dermatomes 0ystein P. Nygaard, MD,t and Svein Ivar Mellgren, MD, PhDt SPINE Volume 23, Number 3, pp 348-353 C 1998, Lippincott-Raven Publishers added comment by olmarker

28. effects of nucleus pulposus application on spinal nerve root morphology and function G. Byröd, B. Rydevik, C. Nordborg, K. Olmarker European Spine Journal December 1998, Volume 7, Issue 6, pp 445-449 Early

29. Nociceptor sensitization in pain pathogenesis Michael S Gold \& Gerald F Gebhart nature medicine focus on pain REVIEW

30. A POSSIBLE NEURONAL MECHANISM UNDERLYING REFERRED GROIN PAIN IN LOWER LUMBAR DISC DISEASES H. Sameda, MD, PhD, Orthopaedic Surgeon1; Y. Takahashi, MD, PhD, Orthopaedic Surgeon1; K. Takahashi, MD, PhD, Lecturer1; T. Chiba, MD, PhD, Professor of Anatomy2; S. Ohtori, MD, PhD, Orthopaedic Surgeon1; and H. Moriya, MD, PhD, Professor of Orthopaedics1 from JBJS british J Bone Joint Surg Br May 2003 vol. 85-B no. 4 600-603

31. Diagnosis and treatment of sciatica B W Koes, $1 \mathrm{M} \mathrm{W}$ van Tulder, $2 \mathrm{~W}$ C Peul 3 Clinical review BMJ 2007;334:1313-7

32. The LANSS Pain Scale: the Leeds assessment of neuropathic symptoms and signs. Bennett M. Pain. 2001 May;92(1-2):147-57.

33. Peroneal neuropathy misdiagnosed as L5 radiculopathy: a case report Michael D Reife12 and Christopher M Coulis345 Chiropractic \& Manual Therapies 2013, 21:12 http://www.chiromt.com/content/21/1/12 
34. A prospective study of centralization of lumbar and referred pain. A predictor of symptomatic discs and anular competence. Donelson R1, Aprill C, Medcalf R, Grant W. Spine (Phila Pa 1976). 1997 May 15;22(10):1115-22.

35. Stereoscopic Structure of Sensory Nerve Fibers in the Lumbar Spine and Related Tissues Yuzuru Takahashi, MD, PhD, et al. SPINE Volume 28, Number 9, pp 871-880 @2003, Lippincott Williams \& Wilkins, Inc.

36. Consistence and discrepancy of neuropathic pain screening tools DN4 and ID-Pain. Padua L, Briani C, Truini A et.al. Neurol Sci. 2012 Mar 21.

37. Assessment of nerve involvement in the lumbar spine: agreement between magnetic resonance imaging, physical examination and pain drawing findings Bo C Bertilson, 1 Eva Brosjö,2 Hans Billing,2 and Lars-Erik Strender1 BMC Musculoskelet Disord. 2010; 11: 202.

38. Dermatomes and dogma V Apok,1 N T Gurusinghe,23Dr H C A Emsley,2 J D Mitchell, DERMATOMES AND THEIR SIGNIFICANCE 3 H C A Emsley Pract Neurol 2011; 11: 100-105

39. An evidence-based approach to human dermatomes. Lee MW, McPhee RW, Stringer MD. Clinical Anatomy (New York, N.Y.) [2008, 21(5):363-73] IJ678

40. Symptom provocation of fluoroscopically guided cervical nerve root stimulation. Are dynatomal maps identical to dermatomal maps? Spine (Phila Pa 1976). 1998 Oct 15;23(20):2235-42. Slipman CW, Plastaras CT, Palmitier RA, et al

41. Pain patterns and descriptions in patients with radicular pain: Does the pain necessarily follow a specific dermatome? Donald R Murphy 1,2,3, Eric L Hurwitz4, Jonathan K Gerrard5 and Ronald Clary6 Chiropractic \& Osteopathy 2009, 17:9 doi:10.1186/1746-1340-17-9

42. DERMATOME PATTERNS IN THE EXTREMITIES JAMA. 1949;140(10):877-878. doi:10.1001/jama.1949.02900450027010.

43. Symptoms and signs of sciatica and their relation to the localization of the lumbar disc herniation. Kortelainen P, Puranen J, Koivisto E, Lähde S. Spine (Phila Pa 1976). 1985 Jan-Feb;10(1):88-92.

44. Study on dermatomes by means of selective lumbar spinal nerve block. Nitta H1, Tajima T, Sugiyama H, Moriyama A. Spine (Phila Pa 1976). 1993 Oct 1;18(13):1782-6.

45. Value of perineural edema/inflammation detected by fat saturation sequences in lumbar magnetic resonance imaging of patients with unilateral sciatica. Sirvanci M, Kara B, Duran C, Ozturk E, Karatoprak O, Onat L, Ulusoy OL, Mutlu A Acta Radiologica (Stockholm, Sweden : 1987) [2009, 50(2):205-11]

46. CT scanning of lumbar discography. A useful diagnostic adjunct. McCutcheon ME, Thompson WC 3rd. Spine (Phila Pa 1976). 1986 Apr;11(3):257-9.

47. Value of 3D MR Lumbosacral Radiculography in the Diagnosis of Symptomatic Chemical Radiculitis AJNR Am J Neuroradiol 33:529 -34 Mar 2012 www.ajnr.org

48. High-resolution 3-T MR neurography of the lumbosacral plexus. Soldatos T1, Andreisek G, Thawait GK, Guggenberger R, Williams EH, Carrino JA, Chhabra A. Radiographics. 2013 Jul-Aug;33(4):967-87.

49. Clinical findings and electrodiagnostic testing in 108 consecutive cases of lumbosacral radiculopathy due to herniated disc. Mondelli M1, Aretini A, Arrigucci U, Ginanneschi F, Greco G, Sicurelli F. Neurophysiol Clin. 2013 Oct;43(4):205-15. 
50. Tumor necrosis factor- $\alpha$ levels correlate with postoperative pain severity in lumbar disc hernia patients: opposite clinical effects between tumor necrosis factor receptor 1 and 2. Andrade P, Visser-Vandewalle V, Philippens M, et al. Pain 2011;152:2645-52.

51. Diagnostic imaging for low back pain: advice for high-value health care from the American College of Physicians. Chou R, Qaseem A, Owens DK, Shekelle P. Ann Intern Med 2011;154:181-9. [Erratum, Ann Intern Med 2012;156:71]

52. [Real Help and Red Herrings in Spinal Imaging Dr. Richard Deyo, $\mathrm{n}$ engl $\mathrm{j}$ med 368;11 nejm.org march 14, 2013]

53. Repeatability of measuring sciatic nerve excursion during a modified passive straight leg raise test with ultrasound imaging .Colette Ridehalgh, Ann Moore, Alan Hough Manual Therapy Volume 17, Issue 6, Pages 572-576, December 2012

54. Reliability, validity and diagnostic accuracy of palpation of the sciatic, tibial and common peroneal nerves in the examination of low back related leg pain Jeremy Walsh a, , Toby Hall b, c Manual Therapy 14 (2009) 623-629

55. In-vivo Endoscopic Visualization of Patho-anatomy in Symptomatic Degenerative Conditions of the Lumbar Spine II: Intradiscal, Foraminal, and Central Canal Decompression. Yeung AT1, Gore S. Surg Technol Int. 2011 Dec 1;XXI:299-319.

56. Interrater Reliability of a New Classification System for Patients with Neural Low Back-Related Leg Pain J Man Manip Ther. 2009; 17(2): 109-117. Axel Schäfer, PT,ab Toby M Hall,et al

57. Agreement and correlation between the straight leg raise and slump tests in subjects with leg pain. J Manipulative Physiol Ther. 2009 Mar-Apr;32(3):184-92 Walsh J, Hall T.

58. The sensitivity and specificity of the Slump and the Straight Leg Raising tests in patients with lumbar disc herniation. J Clin Rheumatol. 2008 Apr;14(2):87-91. Majlesi J, Togay H, Unalan H, et al

59. DATA on distal block for sciatica diagnosis and treatment. GORE s. data presented at wcmisst Hawaii 2008 meet, awarded best clinical study award.

60. Comprehensive review of neurophysiologic basis and diagnostic interventions in managing chronic spinal pain. Manchikanti L1, Boswell MV, Singh V, Derby R, Fellows B, Falco FJ, Datta S, Smith HS, Hirsch JA. Pain Physician. 2009 JulAug;12(4):E71-120.

61. Efficacy of lumbosacral transforaminal epidural steroid injections: a systematic review. PM R. 2009 Jul;1(7):657-68.Roberts ST1, Willick SE, Rho ME, Rittenberg JD.

62. neural blockade for diagnosis and prognosis a review. Anesthsiology 1997 p216-41.hogan and abram

63. Value of Diagnostic Lumbar Selective Nerve Root Block: A Prospective Controlled Study AJNR Am J Neuroradiol 29:1017-23 May 2008 www.ajnr.org

64. The risks of epidural and transforaminal steroid injections in the Spine: Commentary and a comprehensive review of the literature. Surg Neurol Int 2013;4:S74-93. Epstein NE.

65. The problem with diagnostic selective nerve root blocks. Spine (Phila Pa 1976). 2012 Nov 15;37(24):1991-3. Shah RV.

66. Diagnostic utility of selective nerve root blocks in the diagnosis of lumbosacral radicular pain: systematic review and update of current evidence. Datta S1, Manchikanti L, Falco FJ, Calodney AK, Atluri S, Benyamin RM, Buenaventura RM, Cohen SP. Pain Physician. 2013 Apr;16(2 Suppl):SE97-124. 
67. The diagnostic utility and cost-effectiveness of selective nerve root blocks in patients considered for lumbar decompression surgery: a systematic review and economic model. Beynon R1, Hawkins J, Laing R, Higgins N, Whiting P, Jameson C, Sterne JA, Vergara P, Hollingworth W. Health Technol Assess. 2013 May;17(19):1-88, v-vi.

68. Relief of pain and paraesthesiae by nerve block distal to a lesion. KIBLER RF, NATHAN PW. J Neurol Neurosurg Psychiatry. 1960 May;23:91-8.

69. Relief of sciatic nerve pain by sciatic nerve block clinical reports [anne Xavier Anaesth analgesia 1988 1177-80] highlights peripheral inputs as a cause of pain.

70. pain mechanisms in sciatica. Anesthesia analgesia $19881135-7$ abram.

71. Common peroneal nerve block for sciatica. Tajiri K1, Takahashi K, Ikeda K, Tomita K. Clin Orthop Relat Res. 1998 Feb;(347):203-7.

72. The accuracy of the physical examination for the diagnosis of midlumbar and low lumbar nerve root impingement. Suri P, Rainville J, Katz JN, Jouve C, Hartigan C, Limke J, Pena E, Li L, Swaim B, Hunter DJ. Spine (Phila Pa 1976). 2011 Jan 1;36(1):63-73.

73. General principles of diagnostic testing as related to painful lumbar spine disorders: a critical appraisal of current diagnostic techniques. Saal JS. Spine (Phila Pa 1976). 2002 Nov 15;27(22):2538-45; discussion 2546.

74. The tissue origin of low back pain and sciatica: a report of pain response to tissue stimulation during operations on the lumbar spine using local anesthesia. Kuslich SD, Ulstrom CL, Michael CJ. Orthop Clin North Am. 1991 Apr;22(2):181-7.

75. Effects of Nucleus Pulposus on Nerve Root Neural Activity, Mechanosensitivity, Axonal Morphology, and Sodium Channel Expression Spine 2004;29:17-25.

76. Clinical evidence of chemical radiculopathy. Slipman CW, Isaac Z, Lenrow DA, Chou LH, Gilchrist RV, Vresilovic EJ. Pain Physician. 2002 Jul;5(3):260-5

77. Local application of disc-related cytokines on spinal nerve roots. Aoki Y, Rydevik B, Kikuchi S, Olmarker K. Spine (Phila Pa 1976). 2002 Aug 1;27(15):1614-7.

78. Mechanical and biochemical injury of spinal nerve roots: a morphological and neurophysiological study. Cornefjord M, Olmarker K, Rydevik R, Nordborg C. Eur Spine J. 1996;5(3):187-92.

79. Effects of anulus fibrosus and experimentally degenerated nucleus pulposus on nerve root conduction velocity: relevance of previous experimental investigations using normal nucleus pulposus. Iwabuchi M, Rydevik B, Kikuchi S, Olmarker K. Spine (Phila Pa 1976). 2001 Aug 1;26(15):1651-5.

80. Adjacent double-nerve root contributions in unilateral lumbar radiculopathy. Bartynski WS, Kang MD, Rothfus WE. AJNR Am J Neuroradiol. 2010 Feb;31(2):327-33. Epub 2009 Oct 15.

81. Effects of lidocaine on blood flow and endoneurial fluid pressure in a rat model of herniated nucleus pulposus. Onda A, Yabuki S, Kikuchi S, Satoh K, Myers RR. Spine (Phila Pa 1976). 2001 Oct 15;26(20):2186-91; discussion 2191-2.

82. Effect of local and intravenous lidocaine on ongoing activity in injured afferent nerve fibers. Kirillova I, Teliban A, Gorodetskaya N, Grossmann L, Bartsch F, Rausch VH, Struck M, Tode J, Baron R, Jänig W Pain. 2011 Jul;152(7):1562-71. Epub 2011 Apr 6

83. Mechanism-based Classification of Pain for Physical Therapy Management in Palliative care: A Clinical Commentary. Kumar SP, Saha S. Indian J Palliat Care. 2011 Jan;17(1):80-6. 
84. The reliability of clinical judgments and criteria associated with mechanisms-based classifications of pain in patients with low back pain disorders: a preliminary reliability study. Smart KM, Curley A, Blake C, Staines A, Doody C. J Man Manip Ther. 2010 Jun;18(2):102-10.

\section{Disclosures}

Dr. Gore is a consultant to Karl Storz Germany. Sunil Nadkarni declares no financial disclosures.

\section{Corresponding Author}

Satishchandra Gore, MS, FABMISS, Prime Surgical Center, Damle Path, Pune, India 411004; email: yesgore@yahoo.co.in.

Copyright (C) 2014 ISASS - International Society for the Advancement of Spine Surgery. To see more or order reprints or permissions, see http://ijssurgery.com. 